Article

\title{
Seed Pretreatment and Foliar Application of Proline Regulate Morphological, Physio-Biochemical Processes and Activity of Antioxidant Enzymes in Plants of Two Cultivars of Quinoa (Chenopodium quinoa Willd.)
}

\author{
Hira Yaqoob ${ }^{1}$, Nudrat A. Akram ${ }^{1, *}$, Samrah Iftikhar ${ }^{1}$, Muhammad Ashraf ${ }^{2}$, Noman Khalid ${ }^{1}$, \\ Muhammad Sadiq ${ }^{1}$, Mohammed Nasser Alyemeni ${ }^{3}$, Leonard Wijaya ${ }^{3}$ and Parvaiz Ahmad ${ }^{3,4, * \mathbb{C}}$ \\ 1 Department of Botany, Government College University, Faisalabad 38040, Pakistan; \\ hira.yaqoob@gmail.com (H.Y.); samrahiftikhar@gmail.com (S.I.); nomankhalid@gmail.com (N.K.); \\ sadiq.muhammad@gmail.com (M.S.) \\ 2 University of Agriculture, Faisalabad 38040, Pakistan; ashrafbot@yahoo.com \\ 3 Botany and Microbiology Department, College of Science, King Saud University, Riyadh 11451, \\ Saudi Arabia; mnyemeni@ksu.edu.sa (M.N.A.); leon077@gmail.com (L.W.) \\ 4 Department of Botany, S.P. College, Srinagar 190001, Jammu and Kashmir, India \\ * Correspondence: nudrataauaf@yahoo.com (N.A.A.); parvaizbot@yahoo.com (P.A.); \\ Tel.: +923228780051 (N.A.A.)
}

Received: 25 September 2019; Accepted: 7 December 2019; Published: 10 December 2019

\begin{abstract}
In the current study, the effects of exogenously applied proline (25 and $50 \mathrm{mM}$ ) and low-temperature treatment were examined on the physiochemical parameters in the plants of two cultivars $\left(\mathrm{V}_{1}\right.$ and $\left.\mathrm{V}_{2}\right)$ of quinoa (Chenopodium quinoa Willd.). The seeds were also exposed to chilling stress at $4{ }^{\circ} \mathrm{C}$ before sowing. Plants raised from the seeds treated with low temperature showed reduced plant growth and contents of chlorophyll and carotenoids, but they had significantly increased contents of malondialdehyde, proline, ascorbic acid, total free amino acids, total soluble sugars, and total phenolics, as well as the activity of the peroxidase (POD) enzyme. Cold stress applied to seeds remained almost ineffective in terms of bringing about changes in plant root, hydrogen peroxide, glycine betaine and activities of superoxide dismutase (SOD), and catalase (CAT) enzymes. The exogenous application of proline significantly increased plant growth, the contents of chlorophyll, carotenoids, proline, ascorbic acid, total free amino acids, phenolics, and total soluble sugars, as well as the activities of SOD, POD, and CAT, but it decreased malondialdehyde content. Overall, foliar application of proline was better than the seed treatment in improving root dry weight, root length, chlorophyll $a$, carotenoids, glycine betaine, ascorbic acid and superoxide dismutase activity, whereas seed pre-treatment with proline was effective in improving shoot dry weight, shoot length, hydrogen peroxide, malondialdehyde, and peroxidase activity in both quinoa cultivars.
\end{abstract}

Keywords: antioxidants; low temperature stress; proline; Quinoa (Chenopodium quinoa)

\section{Introduction}

Abiotic factors include cold, heat, drought, salinity, toxicity of heavy metals, etc. that severely influence plant growth and development, thereby decreasing overall plant yield. They also act as growth-limiting factors by producing reactive oxygen species (ROS), ultimately causing oxidative stress [1-3]. This oxidative stress triggers metabolic disorders and causes a loss of organelle functions and cell injury, thereby leading to cell death $[4,5]$. Cold stress is one of the major abiotic stresses that directly influences as well as reduces plant growth and yield [6-8]. It has been estimated that up 
to $25 \%$ of crop yield losses occur due to chilling stress $[9,10]$. To cope with unfavorable situations, plants have evolved molecular as well as physio-biochemical adaptations [11]. In response to chilling stress, a number of defensive processes activate within the cells such as cell structure modification, accumulation of antioxidants and osmoprotectants, modification in gene expression, and the generation of anti-freezing proteins $[12,13]$.

Under abiotic stresses, osmoprotectants such as sugars, polyols and proline accumulate which can effectively enhance stress tolerance in plants. They can be utilized as molecular markers for breeding for enhanced stress tolerance [14,15]. Of a variety of osmoprotectants, proline is considered as a vital biomolecule and has been reported to play a significant role in various stresses, both abiotic and biotic [16]. Proline also acts as a potential scavenger of reactive oxygen species [17]. For maintaining optimal cellular levels of proline, foliar application of proline is an effective option [18].

Proline also facilitates the transduction of signals, acts as a substitute of carbon and nitrogen, decreases the membranous injury carried out by ROS species, and maintains protein complexes and DNA, thus performing a number of imperative functions in plants subjected to stressful cues [19]. Stress resistance in plants also increases by the exogenous application of proline, which acts as a cryo-protectant or osmoprotectant [20].

Under stress conditions, proline constitutes approximately $80 \%$ of free amino acids in plants [21]. Proline not only participates in the synthesis of primary metabolites, but it also transports metabolites at the point of growth and development [22,23]. Proline is believed to help in oxidative phosphorylation in mitochondria and triggers ATP synthesis as a stress recovery mechanism [24]. Proline shows stress ameliorative effects in different crops; e.g., maize [25], tobacco [26], wheat [27], and olive [28].

Of various cereal crops, Chenopodium quinoa has an outstanding food quality as well as high resistance to abiotic stresses $[29,30]$. Due to this fact, the cultivation of this crop has spanned the globe $[31,32]$. It can be cultivated in the Andean regions with nutritionally poor lands, and native people there use it as a staple food [32,33]. Seeds of quinoa are a rich source of minerals (magnesium, zinc, lithium, copper, iron and calcium), vitamins (A, $B_{2}$ and $E$ ), basic amino acids, fatty acids and carbohydrates [34,35], which are medicinally very important [29]. Seeds are gluten free and can be used to prepare gluten-free food for celiac patients [29].

We hypothesized that the exogenous application of proline or low temperature could effectively improve key morpho-physiological and biochemical attributes in Chenopodium quinoa plants, which in turn could improve growth. Thus, in the present study, the role of proline and low temperature as a pre-sowing seed treatment and proline foliar application in promoting growth, some biochemical processes, and the oxidative defense system were assessed in quinoa plants.

\section{Materials and Methods}

\subsection{Experimental Design and Treatment}

The research was carried out to investigate the effect of proline applied as a seed treatment and through leaves to quinoa (Chenopodium quinoa) plants raised from seeds treated with low-temperature stress. An experiment was conducted under natural climatic conditions during October-December, 2016 at the Department of Botany, GC University Faisalabad, Pakistan. Seeds of two different quinoa cultivars, namely $\mathrm{V}_{1}$ and $\mathrm{V}_{2}$ (with different genetic makeup), were provided by the Crop Physiology Department, University of Agriculture, Faisalabad, Pakistan. For cold treatment, the seeds were taken in Petri plates and kept at $4{ }^{\circ} \mathrm{C}$ in a refrigerator for $20 \mathrm{~h}$ to induce chilling stress. After that, the seeds were treated with varying effective concentrations $(0,25$ and $50 \mathrm{mM})$ of proline for $15 \mathrm{~h}$ following Kamran et al. [36]. The seeds so primed were sown in disposable plastic glasses each containing $0.407 \mathrm{~kg}$ sandy loam soil. The experiment was arranged in a completely randomized design with three replicates per treatment. Thinning was done after three weeks of germination to maintain five plants of equal size per pot. During the course of the experiment, the average temperature of $28^{\circ} \mathrm{C}$ (day) and $20{ }^{\circ} \mathrm{C}$ (night), relative humidity of $61 \%$ and day-length of $7.5 \mathrm{~h}$ were recorded. After one month of seed germination, proline was applied as a foliar spray using an ordinary sprayer with a sharp nozzle. 
Three concentrations $(0,25$ and $50 \mathrm{mM})$ of proline were prepared in distilled water containing $0.1 \%$ Tween-20 as a surfactant. To the chilled but non-primed seedlings, proline was applied as a foliar spray. After two weeks of exogenous application, the plant samples were collected to measure the following parameters.

\subsection{Plant Growth}

Two plants were harvested from each replication at 40 days after seed germination and washed carefully with distilled water to dislodge the soil particles. The fresh weights of all shoot and root samples were precisely measured; then, all samples were placed in an oven at $80{ }^{\circ} \mathrm{C}$ for $72 \mathrm{~h}$, and then the dry weights were recorded.

\subsection{Chlorophyll Contents}

Fresh leaf material (each $0.5 \mathrm{~g}$ ) was extracted with $80 \%$ acetone in a pestle and mortar followed by centrifugation to obtain the supernatant [37]. The optical density of the supernatant was read at 645 and $663 \mathrm{~nm}$ using a spectrophotometer.

\subsection{Hydrogen Peroxide $\left(\mathrm{H}_{2} \mathrm{O}_{2}\right)$ Contents}

The leaf material $(0.25 \mathrm{~g})$ was triturated using a pestle and mortar containing $5 \mathrm{~mL}$ of $0.1 \%$ trichloroacetic acid following the method of Velikova, et al. [38]. The homogenized material was centrifuged for $15 \mathrm{~min}$ at $12,000 \times \mathrm{g}$. To $0.5 \mathrm{~mL}$ of the supernatant, $1 \mathrm{~mL}$ of $1 \mathrm{M}$ potassium iodide and $0.5 \mathrm{~mL}$ of potassium phosphate buffer were added, and the mixture was shaken vigorously. The absorbance of the mixture was read at $390 \mathrm{~nm}$ using a spectrophotometer.

\subsection{Malondialdehyde (MDA) Contents}

The protocol of Heath and Packer [39] was employed to determine the contents of MDA. Fresh leaf material (each $0.25 \mathrm{~g}$ ) was ground with $2.5 \mathrm{~mL}$ of $5 \%(w / v)$ trichloroacetic acid (TCA). The mixture was centrifuged at $12,000 \times g$ for $15 \mathrm{~min}$. Then, $1 \mathrm{~mL}$ of the supernatant and $4 \mathrm{~mL}$ of thiobarbituric acid (TBA) were mixed. The mixture was placed in a water bath at $95{ }^{\circ} \mathrm{C}$ for $30 \mathrm{~min}$, the reaction mixture was chilled, and the absorbance was read at 532 and $600 \mathrm{~nm}$ using a spectrophotometer.

\subsection{Free Proline Contents}

The leaf samples (each $0.25 \mathrm{~g}$ ) were ground each in $5 \mathrm{~mL}$ of sulfosalicylic acid following the method of Bates, et al. [40]. To $2.0 \mathrm{~mL}$ of plant extract, $2 \mathrm{~mL}$ of glacial acetic acid and $2.0 \mathrm{~mL}$ of acidic ninhydrin $(2 \mathrm{~mL})$ were added. The mixture was shaken vigorously and placed in a water bath for one $\mathrm{h}$ at $75{ }^{\circ} \mathrm{C}$. To stop the reaction, the mixture was placed on ice. The proline contents were obtained from the chromophore layer by adding $4 \mathrm{~mL}$ of toluene to the reaction mixture. The toluene containing the chromophore was isolated and the absorbance read at $520 \mathrm{~nm}$.

\subsection{Ascorbic Acid}

The Mukherjee and Choudhuri [41] protocol was employed to determine the contents of ascorbic acid. Plant material was homogenized with 6\% TCA solution and filtered. An aliquot ( $2 \mathrm{~mL})$ of the filtrate was mixed with $1 \mathrm{~mL}$ dinitrophenyl hydrazine in $9 \mathrm{~N} \mathrm{H}_{2} \mathrm{SO}_{4}(2 \%)$, and then a droplet of thiourea (prepared in $70 \%$ ethanol) was added to it. The samples were placed in a water bath for 15 min and then cooled at room temperature. After that, $2.5 \mathrm{~mL}$ of $80 \%$ sulfuric acid was added to the reaction mixture and the optical density (OD)was measured at $530 \mathrm{~nm}$ using a spectrophotometer.

\subsection{Antioxidant Enzymes}

To obtain plant crude extract, fresh ice-cold leaves (each $0.25 \mathrm{~g}$ ) were homogenized in $10 \mathrm{~mL}$ of $50 \mathrm{mM}$ of potassium phosphate buffer; then, the mixture was centrifuged and the supernatant preserved in Eppendorf tubes in a freezer to appraise the activities of the following enzymes. 


\subsection{Catalase (CAT) and Peroxidase (POD)}

The activities of CAT and POD enzymes were determined following the protocol of Chance and Maehly [42]. Crude enzyme extract $(100 \mu \mathrm{L}), 1.9 \mathrm{~mL}$ of $50 \mathrm{mM}$ buffer $(1 \mathrm{~mL})$ and $1 \mathrm{~mL}$ of $\mathrm{H}_{2} \mathrm{O}_{2}(40 \mathrm{mM})$ were mixed. The variation in absorbance of the reaction solution was read subsequently after every 20 seconds for $3 \mathrm{~min}$ at $240 \mathrm{~nm}$ using a spectrophotometer. To determine the peroxidase activity, the reaction mixture contained distilled water, $250 \mu \mathrm{L}$ phosphate buffer $(50 \mathrm{mM}), 100 \mu \mathrm{L} \mathrm{H}_{2} \mathrm{O}_{2}, 20 \mathrm{mM}$ guaicol $(100 \mu \mathrm{L})$ and the enzyme extract $(50 \mu \mathrm{L})$. The change in the enzyme activity was noted after every 20 seconds for $3 \mathrm{~min}$ at $470 \mathrm{~nm}$ using a spectrophotometer. One unit of CAT and POD activity was defined as 0.01 absorbance change per min.

\subsection{Superoxide Dismutase (SOD)}

The protocol of Giannopolitis and Ries [43] was followed to determine the SOD activity. The reaction mixture for the SOD had $50 \mathrm{mM}$ phosphate buffer, $13 \mathrm{mM}$ methionine, $1.3 \mu \mathrm{M}$ riboflavin, distilled water, and $50 \mu \mathrm{L}$ supernatant. The reaction mixture was placed under a fluorescent lamp for $15 \mathrm{~min}$ and the absorbance read at $560 \mathrm{~nm}$ using a spectrophotometer. One unit of SOD activity was defined as the amount required to inhibit the photo-reduction of nitroblue tetrazolium (NBT) by $50 \%$.

\subsection{Total Soluble Sugars}

For the estimation of total soluble sugars, leaf material (each $0.5 \mathrm{~g}$ ) was ground with $5 \mathrm{~mL}$ of $80 \%$ ethanol in a pestle and mortar. The samples were subjected to shaking for $1 \mathrm{~h}$ at $60^{\circ} \mathrm{C}$. To one $\mathrm{mL}$ of the supernatant, $3 \mathrm{~mL}$ of anthrone reagent (dissolved $150 \mathrm{mg}$ anthrone in $72 \%$ freshly prepared $\mathrm{H}_{2} \mathrm{SO}_{4}$ ) was added; then, the mixture was heated for $10 \mathrm{~min}$, the samples cooled down at room temperature for $20 \mathrm{~min}$, and the absorbance read at $625 \mathrm{~nm}$ using a spectrophotometer following Yemm and Willis [44].

\subsection{Total Phenolics}

Leaf samples (each $0.5 \mathrm{~g}$ ) were homogenized, each in $10 \mathrm{~mL}$ of $80 \%$ acetone. The mixture was centrifuged at $15,000 \times \mathrm{g}$ at $4{ }^{\circ} \mathrm{C}$ for $15 \mathrm{~min}$. To $0.1 \mathrm{~mL}$ of plant extract, $2 \mathrm{~mL}$ of distilled water and $0.5 \mathrm{~mL}$ of Folin-Ciocalteau's reagent were added. The samples were then shaken vigorously. By adding $2.5 \mathrm{~mL}$ of $20 \%$ sodium carbonate to each sample, all samples were vortexed for 10 seconds and their final volume was raised to $7.5 \mathrm{~mL}$ by adding distilled water. After $20 \mathrm{~min}$, the absorbance of all samples was read at $750 \mathrm{~nm}$ following Julkunen-Tiitto [45].

\subsection{Free Amino Acids}

The leaf sample (each $0.5 \mathrm{~g}$ ) was ground in $10 \mathrm{~mL}$ of potassium phosphate buffer. The mixture was centrifuged at $12,000 \times g$ at $4{ }^{\circ} \mathrm{C}$. To $1 \mathrm{~mL}$ of the supernatant, $1 \mathrm{~mL}$ of ninhydrin $(2 \%)$ and $1 \mathrm{~mL}$ of pyridine $(10 \%)$ were added, and the mixture was placed in a water bath for $30 \mathrm{~min}$. After cooling all samples, they were read at $750 \mathrm{~nm}$ using a spectrophotometer as described by Hamilton, et al. [46].

\subsection{Statistical Analysis}

The experiment was arranged in a completely randomized design with three factors (cultivars, cold stress, exogenous application) with three replicates. After working out the analysis of variance of data for each parameter, the means were compared at a $0.05 \%$ level with the least significant difference (LSD) test.

\section{Results}

Seeds of two quinoa cultivars $\left(\mathrm{V}_{1}\right.$ and $\left.\mathrm{V}_{2}\right)$ treated with cold stress at $4{ }^{\circ} \mathrm{C}$ for $20 \mathrm{~h}$ were grown for 40 days under natural growth conditions (Supplementary Figure S1). Data showed that the shoot fresh and dry weights of the plants of both quinoa cultivars raised from cold-stressed seeds were significantly $(P \leq 0.001)$ reduced as compared to those of the plants raised from non-treated seeds. An exogenous 
application (pre-sowing) of proline ( 25 and $50 \mathrm{mM}$ ) was useful in improving the shoot fresh as well as dry weights of both quinoa cultivars under stress and non-stress conditions. The cultivar $V_{1}$ had greater shoot fresh and dry weights than those of $V_{2}$ under cold stress (Figure 1, Tables 1 and 2).

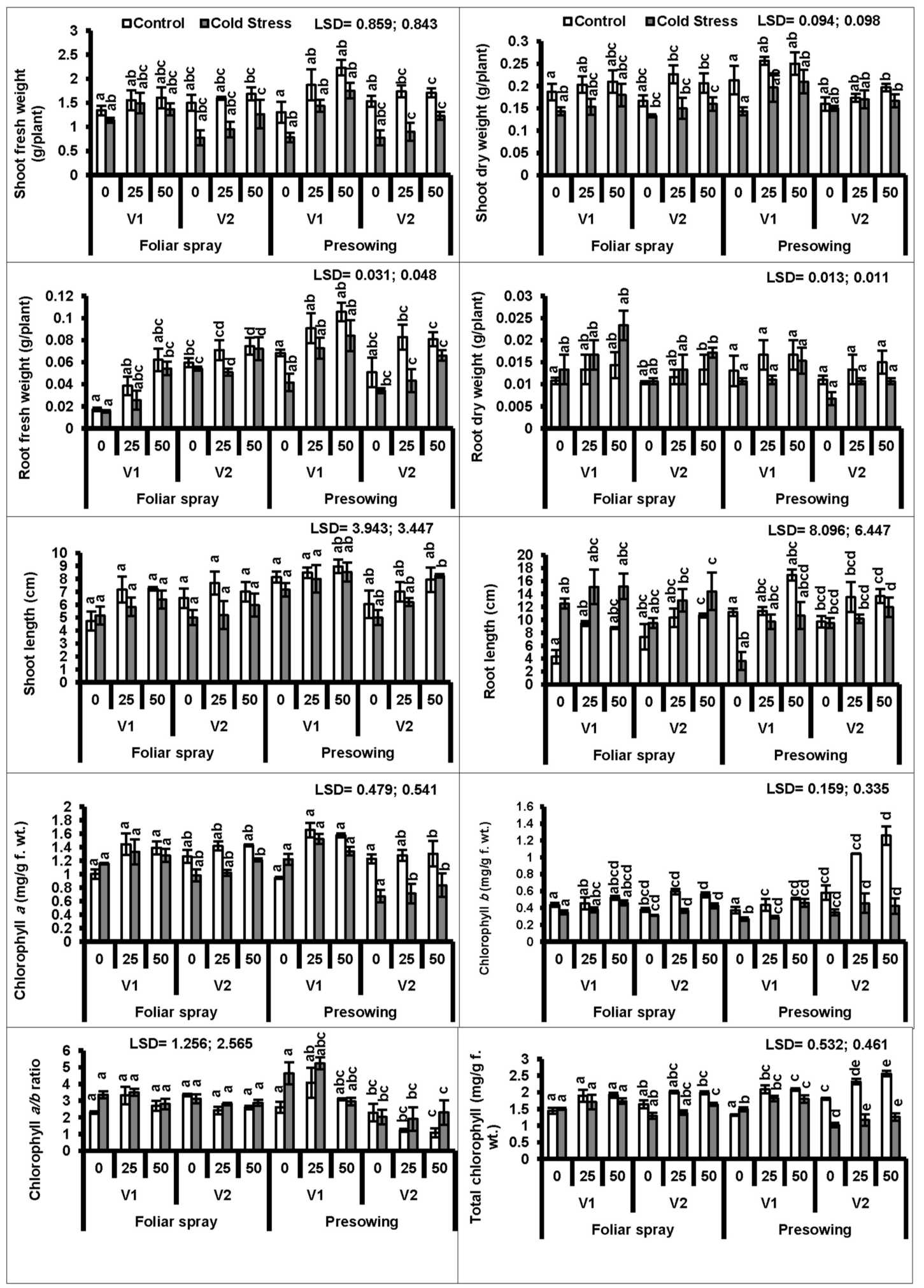

Figure 1. Shoot fresh and dry weights, root fresh and dry weights, shoot and root length, chlorophyll pigments ( $\mathrm{a}$ and $\mathrm{b}$ ), chlorophyll $\mathrm{a} / \mathrm{b}$ ratio and total chlorophyll of quinoa plants subjected to chilling stress (mean \pm S.E.); Different letters $(a-c)$ show significant difference among treatments. $f . w t$; fresh weight. 
Table 1. Analyses of variance data for different physio-biochemical attributes of chilling stressed quinoa (Chenopodium quinoa) plants subjected to varying (25 and $50 \mathrm{mM}$ ) levels of foliar-applied proline. SOD: superoxide dismutase.

\begin{tabular}{|c|c|c|c|c|c|c|c|}
\hline Source of Variation & df & Shoot Fresh Weight & Shoot Dry Weight & Root Fresh Weight & Root Dry Weight & Shoot Length & Root Length \\
\hline Cultivars (Cv) & 1 & $0.132 \mathrm{~ns}$ & $0.0002 \mathrm{~ns}$ & $0.0005 .8 \mathrm{~ns}$ & $0.00005 \mathrm{~ns}$ & $0.16 \mathrm{~ns}$ & $0.0011 \mathrm{~ns}$ \\
\hline Chilling Stress (CS) & 1 & $1.311^{* * *}$ & $0.0196^{* * *}$ & $0.0072^{* * *}$ & 0.0001 .1 * & $11.33 *$ & $207.36^{* * *}$ \\
\hline Foliar Spray (FS) & 2 & $0.273 \mathrm{~ns}$ & $0.0034 \mathrm{~ns}$ & $0.0015^{* * *}$ & $0.0043 *$ & $5.94 \mathrm{~ns}$ & $54.5^{* *}$ \\
\hline $\mathrm{Cv} \times \mathrm{CS}$ & 1 & $0.409 *$ & $0.00024 \mathrm{~ns}$ & $0.00054 \mathrm{~ns}$ & $0.00002 \mathrm{~ns}$ & $2.66 \mathrm{~ns}$ & $34.41 *$ \\
\hline $\mathrm{Cv} \times \mathrm{FS}$ & 2 & $0.044 \mathrm{~ns}$ & $0.0005 .5 \mathrm{~ns}$ & $0.000009 \mathrm{~ns}$ & $0.00003 \mathrm{~ns}$ & $1.05 \mathrm{~ns}$ & $1.02 \mathrm{~ns}$ \\
\hline $\mathrm{CS} \times \mathrm{FS}$ & 2 & $0.014 \mathrm{~ns}$ & $0.0006 \mathrm{~ns}$ & $0.00002 \mathrm{~ns}$ & $0.00002 \mathrm{~ns}$ & $1.48 \mathrm{~ns}$ & $0.955 \mathrm{~ns}$ \\
\hline $\mathrm{Cv} \times \mathrm{CS} \times \mathrm{FS}$ & 2 & $0.036 \mathrm{~ns}$ & $0.0002 .6 \mathrm{~ns}$ & $0.0002 \mathrm{~ns}$ & $0.000002 \mathrm{~ns}$ & $0.564 \mathrm{~ns}$ & $2.62 \mathrm{~ns}$ \\
\hline \multirow[t]{2}{*}{ Error } & 24 & & & 0.0001 & 0.00002 & 1.82 & 7.683 \\
\hline & & Chl. $a$ & Chl. $b$ & Chl. $a / b$ & Total Chl. & Carotenoids & Proline \\
\hline Cultivars (Cv) & 1 & $0.019 \mathrm{~ns}$ & $0.0003 \mathrm{~ns}$ & $0.174 \mathrm{~ns}$ & $0.014 \mathrm{~ns}$ & $0.0001 \mathrm{~ns}$ & $79.59 \mathrm{~ns}$ \\
\hline Chilling Stress (CS) & 1 & $0.232 * *$ & $0.104^{* * *}$ & $0.837^{*}$ & $0.648^{* * *}$ & $0.0876^{* * *}$ & $1070.26^{* * *}$ \\
\hline Foliar Spray (FS) & 2 & $0.184^{* *}$ & $0.045^{* * *}$ & $0.308 \mathrm{~ns}$ & $0.407^{* * *}$ & $0.0245^{* *}$ & $244.5^{* *}$ \\
\hline Cv $\times$ CS & 1 & $0.169 *$ & $0.0097 \mathrm{~ns}$ & $0.193 \mathrm{~ns}$ & $0.26^{* *}$ & $0.001 \mathrm{~ns}$ & $5.93 \mathrm{~ns}$ \\
\hline $\mathrm{Cv} \times \mathrm{FS}$ & 2 & $0.0352 \mathrm{~ns}$ & $0.0093 \mathrm{~ns}$ & $1.107^{* *}$ & $0.0088 \mathrm{~ns}$ & $0.00005 \mathrm{~ns}$ & $15.75 \mathrm{~ns}$ \\
\hline $\mathrm{CS} \times \mathrm{FS}$ & 2 & $0.0269 \mathrm{~ns}$ & $0.0045 \mathrm{~ns}$ & $0.0308 \mathrm{~ns}$ & $0.052 \mathrm{~ns}$ & $0.0039 \mathrm{~ns}$ & $40.36 \mathrm{~ns}$ \\
\hline $\mathrm{Cv} \times \mathrm{CS} \times \mathrm{FS}$ & 2 & $0.0214 \mathrm{~ns}$ & $0.00563 \mathrm{~ns}$ & $0.52 \mathrm{~ns}$ & $0.0165 \mathrm{~ns}$ & $0.000065 \mathrm{~ns}$ & $4.64 \mathrm{~ns}$ \\
\hline \multirow[t]{2}{*}{ Error } & 24 & 0.0269 & 0.0029 & 0.185 & 0.9332 & 0.0033 & 35.04 \\
\hline & & Glycine Betaine & Ascorbic Acid & Hydrogen Peroxide & MDA & Catalase & Peroxidase \\
\hline Cultivars $(\mathrm{Cv})$ & 1 & $6553.30 * * *$ & $4.54 \mathrm{~ns}$ & $1463.05 \mathrm{~ns}$ & $2.9221 \mathrm{e}-4 \mathrm{~ns}$ & $1.24^{* * *}$ & $0.0035 \mathrm{~ns}$ \\
\hline Chilling Stress (CS) & 1 & $288.79 \mathrm{~ns}$ & $80.14^{* * *}$ & $20928.4^{* *}$ & $116.7^{* * *}$ & $0.013 \mathrm{~ns}$ & 0.030 * \\
\hline Foliar Spray (FS) & 2 & $259.63 \mathrm{~ns}$ & $50.41^{* * *}$ & $13452.4^{* *}$ & $24.30^{* * *}$ & 0.059 * & 0.0117 ns \\
\hline $\mathrm{Cv} \times \mathrm{CS}$ & 1 & 2106.43 * & $0.978 \mathrm{~ns}$ & $28.44 \mathrm{~ns}$ & 18.48 * & $0.182 \mathrm{~ns}$ & $0.0087 \mathrm{~ns}$ \\
\hline $\mathrm{Cv} \times \mathrm{FS}$ & 2 & $175.61 \mathrm{~ns}$ & $3.16 \mathrm{~ns}$ & $2696.2 \mathrm{~ns}$ & $1.54 \mathrm{~ns}$ & $0.0033 \mathrm{~ns}$ & $0.0023 \mathrm{~ns}$ \\
\hline CS $x$ FS & 2 & $648.77 \mathrm{~ns}$ & $18.22 \mathrm{~ns}$ & $1711.5 \mathrm{~ns}$ & $0.449 \mathrm{~ns}$ & $0.0124 \mathrm{~ns}$ & $0.0001 \mathrm{~ns}$ \\
\hline $\mathrm{Cv} \times \mathrm{CS} \times \mathrm{FS}$ & 2 & $710.5 \mathrm{~ns}$ & $1.37 \mathrm{~ns}$ & $464.9 \mathrm{~ns}$ & $2.12 \mathrm{~ns}$ & $0.0041 \mathrm{~ns}$ & $0.0009 \mathrm{~ns}$ \\
\hline Error & 24 & 274.01 & 2.414 & 2299.2 & 2.45 & 0.0155 & 0.0042 \\
\hline
\end{tabular}


Table 1. Cont

\begin{tabular}{|c|c|c|c|c|c|}
\hline & & SOD & Total Soluble Sugars & Total Phenolics & Total Free Amino Acids \\
\hline Cultivars $(\mathrm{Cv})$ & 1 & $1.104 \mathrm{~ns}$ & $62.93 *$ & $39.27 \mathrm{~ns}$ & $0.0262 * *$ \\
\hline Chilling Stress (CS) & 1 & $7.02 *$ & $228.01^{* * *}$ & $686.44^{* * *}$ & $0.0162 *$ \\
\hline Foliar Spray (FS) & 2 & $8.909 *$ & $114.3^{* * *}$ & $122.06^{* *}$ & $0.0078 *$ \\
\hline $\mathrm{Cv} \times \mathrm{CS}$ & 1 & $4.867 \mathrm{~ns}$ & $0.694 \mathrm{~ns}$ & $78.91 \mathrm{~ns}$ & $0.0008 \mathrm{~ns}$ \\
\hline $\mathrm{Cv} \times \mathrm{FS}$ & 2 & $2.111 \mathrm{~ns}$ & $17.3 \mathrm{~ns}$ & $15.57 \mathrm{~ns}$ & $0.0001 \mathrm{~ns}$ \\
\hline $\mathrm{CS} \times \mathrm{FS}$ & 2 & $2.91 \mathrm{~ns}$ & $18.73 \mathrm{~ns}$ & $34.22 \mathrm{~ns}$ & $0.0006 \mathrm{~ns}$ \\
\hline $\mathrm{Cv} \times \mathrm{CS} \times \mathrm{FS}$ & 2 & $3.76 \mathrm{~ns}$ & $54.72 \mathrm{~ns}$ & $42.4 \mathrm{~ns}$ & $0.0012 \mathrm{~ns}$ \\
\hline Error & 24 & 1.642 & 11.51 & 18.57 & 0.002 \\
\hline
\end{tabular}

Table 2. Analyses of variance of data for different physio-biochemical attributes of quinoa (Chenopodium quinoa) plants raised from seeds treated with varying (25 and $50 \mathrm{mM}$ ) levels of proline and chilling stress.

\begin{tabular}{|c|c|c|c|c|c|c|c|}
\hline Source of Variation & df & $\begin{array}{l}\text { Shoot Fresh Weight } \\
\text { Shoot Fresh Weight }\end{array}$ & Shoot Dry Weight & Root Fresh Weight & Root Dry Weight & Shoot Length & Root Length \\
\hline Cultivars $(\mathrm{Cv})$ & 1 & $0.558 *$ & $0.016^{* * *}$ & $0.0027^{* *}$ & $0.000006 \mathrm{~ns}$ & $19.65^{* * *}$ & $6.33 \mathrm{~ns}$ \\
\hline Chilling Stress (CS) & 1 & $3.091^{* * *}$ & $0.0113^{* *}$ & $0.0047^{* * *}$ & $0.00001 *$ & $2.89 \mathrm{~ns}$ & $107.8^{* * *}$ \\
\hline Presowing (Pre) & 2 & $1.216^{* * *}$ & 0.0052 * & $0.0038^{* * *}$ & $0.00005 \mathrm{~ns}$ & $10.11 \mathrm{~ns}$ & $69.21^{* * *}$ \\
\hline Cv $\times$ CS & 1 & $0.098 \mathrm{~ns}$ & $0.004 \mathrm{~ns}$ & $0.00005 \mathrm{~ns}$ & $0.0000009 \mathrm{~ns}$ & $0.027 \mathrm{~ns}$ & $26.18 *$ \\
\hline Cv $x$ Pre & 2 & $0.32 *$ & $0.0008 \mathrm{~ns}$ & $0.00006 \mathrm{~ns}$ & $0.000000015 \mathrm{~ns}$ & $1.66 \mathrm{~ns}$ & $8.07 \mathrm{~ns}$ \\
\hline CS $\times$ Pre & 2 & $0.0261 \mathrm{~ns}$ & $0.00005 \mathrm{~ns}$ & $0.000008 \mathrm{~ns}$ & $0.000001 \mathrm{~ns}$ & $0.663 \mathrm{~ns}$ & $2.09 \mathrm{~ns}$ \\
\hline Cv $\times$ CS $\times$ Pre & 2 & $0.0305 \mathrm{~ns}$ & $0.0005 \mathrm{~ns}$ & $0.00002 \mathrm{~ns}$ & $0.0000007 \mathrm{~ns}$ & $0.231 \mathrm{~ns}$ & $15.93 \mathrm{~ns}$ \\
\hline \multirow[t]{2}{*}{ Error } & 24 & 0.0834 & 0.00113 & 0.000002 & 0.00001 & 1.394 & 4.87 \\
\hline & & Chl. $a$ & Chl. $b$ & Chl. a/b & Total Chl. & Carotenoids & Proline \\
\hline Cultivars $(\mathrm{Cv})$ & 1 & $1.23^{* * *}$ & $0.777^{* * *}$ & $34.58^{* * *}$ & $0.0532 \mathrm{~ns}$ & $0.015 *$ & $445.49 *$ \\
\hline Chilling Stress (CS) & 1 & $0.703^{* * *}$ & $0.951^{* * *}$ & $5.49 *$ & $3.29 * * *$ & $0.0006 \mathrm{~ns}$ & $611.02 * *$ \\
\hline Presowing (Pre) & 2 & $0.273 * *$ & $0.223^{* * *}$ & $1.80 \mathrm{~ns}$ & $0.933^{* * *}$ & $0.011^{* *}$ & $909.4^{* * *}$ \\
\hline $\mathrm{Cv} \times \mathrm{CS}$ & 1 & $0.559 * * *$ & $0.462^{* * *}$ & $0.497 \mathrm{~ns}$ & $2.04^{* * *}$ & $0.0054 \mathrm{~ns}$ & $5.33 \mathrm{~ns}$ \\
\hline $\mathrm{Cv} \times$ Pre & 2 & 0.159 * & $0.052 *$ & 2.83 * & $0.039 \mathrm{~ns}$ & $0.00001 \mathrm{~ns}$ & $3.88 \mathrm{~ns}$ \\
\hline CS $x$ Pre & 2 & $0.044 \mathrm{~ns}$ & 0.060 * & $0.136 \mathrm{~ns}$ & $0.205^{* *}$ & $0.000008 \mathrm{~ns}$ & $120.9 \mathrm{~ns}$ \\
\hline Cv $\times$ CS $\times$ Pre & 2 & $0.069 \mathrm{~ns}$ & $0.0809^{* *}$ & $2.46 \mathrm{~ns}$ & $0.0035 \mathrm{~ns}$ & $0.0009 \mathrm{~ns}$ & $262.5^{*}$ \\
\hline Error & 24 & 0.0342 & 0.0131 & 0.772 & 0.024 & 0.002 & 72.99 \\
\hline
\end{tabular}


Table 2. Cont

\begin{tabular}{|c|c|c|c|c|c|c|c|}
\hline & & Glycine Betaine & Ascorbic Acid & Hydrogen Peroxide & MDA & Catalase & Peroxidase \\
\hline Cultivars $(\mathrm{Cv})$ & 1 & $633.52 * *$ & $28.13 * *$ & $1158762.5^{* * *}$ & $26.82 \mathrm{~ns}$ & $0.142 * *$ & $0.00002 \mathrm{~ns}$ \\
\hline Chilling Stress (CS) & 1 & $1386.6^{* * *}$ & $19.09 *$ & $317579.2 * *$ & $266.2 * * *$ & $0.049 \mathrm{~ns}$ & $0.199^{* * *}$ \\
\hline Presowing (Pre) & 2 & $414.3 * *$ & $49.14^{* * *}$ & $67016.8 \mathrm{~ns}$ & $34.64 \mathrm{~ns}$ & $0.050 \mathrm{~ns}$ & $0.0302 * *$ \\
\hline $\mathrm{Cv} \times \mathrm{CS}$ & 1 & $424.9 *$ & $1.98 \mathrm{~ns}$ & $21085.4 \mathrm{~ns}$ & $3.5 \mathrm{~ns}$ & $0.019 \mathrm{~ns}$ & $0.07^{* *}$ \\
\hline $\mathrm{Cv} \times$ Pre & 2 & $6.90 \mathrm{~ns}$ & $1.392 \mathrm{~ns}$ & $10304.3 \mathrm{~ns}$ & $19.68 \mathrm{~ns}$ & $0.0015 \mathrm{~ns}$ & $0.00001 \mathrm{~ns}$ \\
\hline CS $\times$ Pre & 2 & $0.648 \mathrm{~ns}$ & $15.92 *$ & $75875.6 \mathrm{~ns}$ & $8.54 \mathrm{~ns}$ & $0.0047 \mathrm{~ns}$ & $0.0104 \mathrm{~ns}$ \\
\hline $\mathrm{Cv} \times \mathrm{CS} \times$ Pre & 2 & $27.11 \mathrm{~ns}$ & 12.34 * & $7958.5 \mathrm{~ns}$ & $0.75 \mathrm{~ns}$ & $0.0079 \mathrm{~ns}$ & $0.00008 \mathrm{~ns}$ \\
\hline \multirow[t]{2}{*}{ Error } & 24 & 58.01 & 3.04 & 28230.2 & 10.89 & 0.0152 & 0.00008 \\
\hline & & SOD & Total Soluble Sugars & Total Phenolics & Total Free Amino Acids & & \\
\hline Cultivars $(\mathrm{Cv})$ & 1 & $22.35^{* * *}$ & $244.92 *$ & $570.6^{* * *}$ & $0.003 \mathrm{~ns}$ & & \\
\hline Chilling Stress (CS) & 1 & $18.34^{* *}$ & $121.4 \mathrm{~ns}$ & $1348.4^{* * *}$ & $0.022 * *$ & & \\
\hline Presowing (Pre) & 2 & $2.14 \mathrm{~ns}$ & $72.27 \mathrm{~ns}$ & $41.28 \mathrm{~ns}$ & $0.005 \mathrm{~ns}$ & & \\
\hline $\mathrm{Cv} \times \mathrm{CS}$ & 1 & $0.292 \mathrm{~ns}$ & $6.33 \mathrm{~ns}$ & $0.307 \mathrm{~ns}$ & $0.0037 \mathrm{~ns}$ & & \\
\hline Cv $x$ Pre & 2 & $0.782 \mathrm{~ns}$ & $29.98 \mathrm{~ns}$ & $1.195 \mathrm{~ns}$ & $0.00001 \mathrm{~ns}$ & & \\
\hline CS $x$ Pre & 2 & $0.682 \mathrm{~ns}$ & $93.94 \mathrm{~ns}$ & $12.003 \mathrm{~ns}$ & $0.0002 \mathrm{~ns}$ & & \\
\hline $\mathrm{Cv} \times \mathrm{CS} \times$ Pre & 2 & $0.206 \mathrm{~ns}$ & $22.75 \mathrm{~ns}$ & $0.284 \mathrm{~ns}$ & $0.00008 \mathrm{~ns}$ & & \\
\hline Error & 24 & 1.56 & 36.22 & 18.87 & 0.0019 & & \\
\hline
\end{tabular}


Plants of both cultivars raised from the seeds treated with cold stress had significantly lower root fresh and dry weights than those of the plants raised from non-treated seeds. Both modes of application (foliar and seed pre-treatment) of proline showed a significant $(P \leq 0.01)$ enhancing effect only on the root fresh weight. Of both levels of proline, $50 \mathrm{mM}$ proved the most beneficial. The cultivar $\mathrm{V}_{1}$ showed better performance with pre-sowing seed treatments in terms of the root fresh weight (Figure 1).

Plants raised from cold-stressed seeds showed significantly reduced shoot and root lengths $(P \leq 0.001 ; 0.05)$ in both quinoa varieties. However, proline applied via foliar and seed pre-treatment significantly $(P \leq 0.01 ; 0.001)$ increased only the root length. The response of quinoa cultivar $V_{1}$ was more prominent with $50 \mathrm{mM}$ proline applied as a seed pretreatment.

A considerable reduction was observed in chlorophyll $a$, chlorophyll $b$, and the total chlorophyll in the leaves of plants of both quinoa cultivars $\left(\mathrm{V}_{1}\right.$ and $\left.\mathrm{V}_{2}\right)$ rose from cold-stressed seeds. Externally applied (foliar and pre-sowing seed treatment) proline significantly increased both chlorophyll $a$ and $b$ in both quinoa cultivars. In the case of chlorophyll $a$ and $b$ contents, both proline concentrations ( 25 and $50 \mathrm{mM}$ ) as seed treatments were more effective in cultivar $\mathrm{V}_{1}$ compared to $\mathrm{V}_{2}$ (Figure 1).

A prominent reduction $(P \leq 0.001)$ occurred in carotenoid contents, particularly in quinoa plants raised from cold-stressed seeds. The exogenous application (foliar and pre-sowing) of proline considerably $(P \leq 0.01)$ promoted carotenoid contents in both quinoa cultivars. A similar trend was observed in both quinoa cultivars under both types of treatments (Figure 2, Tables 1 and 2).

Low-temperature stress imposed as a pre-sowing treatment caused an enhanced accumulation of endogenous proline in the quinoa plants. The exogenous application of proline significantly $(P \leq 0.001)$ improved the in vivo proline levels in the plants of both cultivars raised from chilling-stressed seeds. The proline applied as a foliar spray was most effective in enhancing endogenous proline levels in both quinoa cultivars (Figure 2).

Intrinsic glycine betaine (GB) contents increased in plants raised from low-temperature stressed seeds. However, seed treatment with proline was effective in enhancing intrinsic GB contents only in cultivar $\mathrm{V}_{2}$ (Figure 2).

Ascorbic acid contents increased considerably in plants of both quinoa cultivars raised from cold-stressed seeds (Tables 1 and 2). Both modes of proline application were effective in promoting AsA concentration in quinoa plants. Both quinoa cultivars showed a similar trend of increasing AsA contents under plants raised from seeds subjected to low temperature (Figure 2).

The quinoa plants raised from cold-treated seeds showed a significant increasing effect on the accumulation of hydrogen peroxide $\left(\mathrm{H}_{2} \mathrm{O}_{2}\right)$ contents. Only the foliage spray of proline application played a significant role in lowering the $\mathrm{H}_{2} \mathrm{O}_{2}$ levels in the quinoa plants (Figure 2).

A high accumulation of malondialdehyde (MDA) was observed in the plants of both quinoa cultivars raised from low-temperature treated seeds. However, the exogenous application of proline through foliar spray $(P \leq 0.001)$ increased the MDA levels in both quinoa cultivars under chilling stress (Figure 2).

The activity of peroxidase enzyme significantly increased $(P \leq 0.001)$, while the activities of catalase (CAT) and superoxide dismutase (SOD) enzymes remained unaffected in quinoa plants developed from seeds treated with cold. The activities of CAT and SOD enzymes were better in quinoa cultivars due to the foliar-applied proline, whereas only the peroxidase activity of both cultivars was affected by the pre-sowing treatment (Figure 2).

In the present study, cold stress applied as a seed pre-treatment significantly $(P \leq 0.001)$ increased the total phenolics in both cultivars of quinoa. The exogenous application of proline as a foliar spray only further increased the total phenolic contents (Figure 3, Tables 1 and 2). 


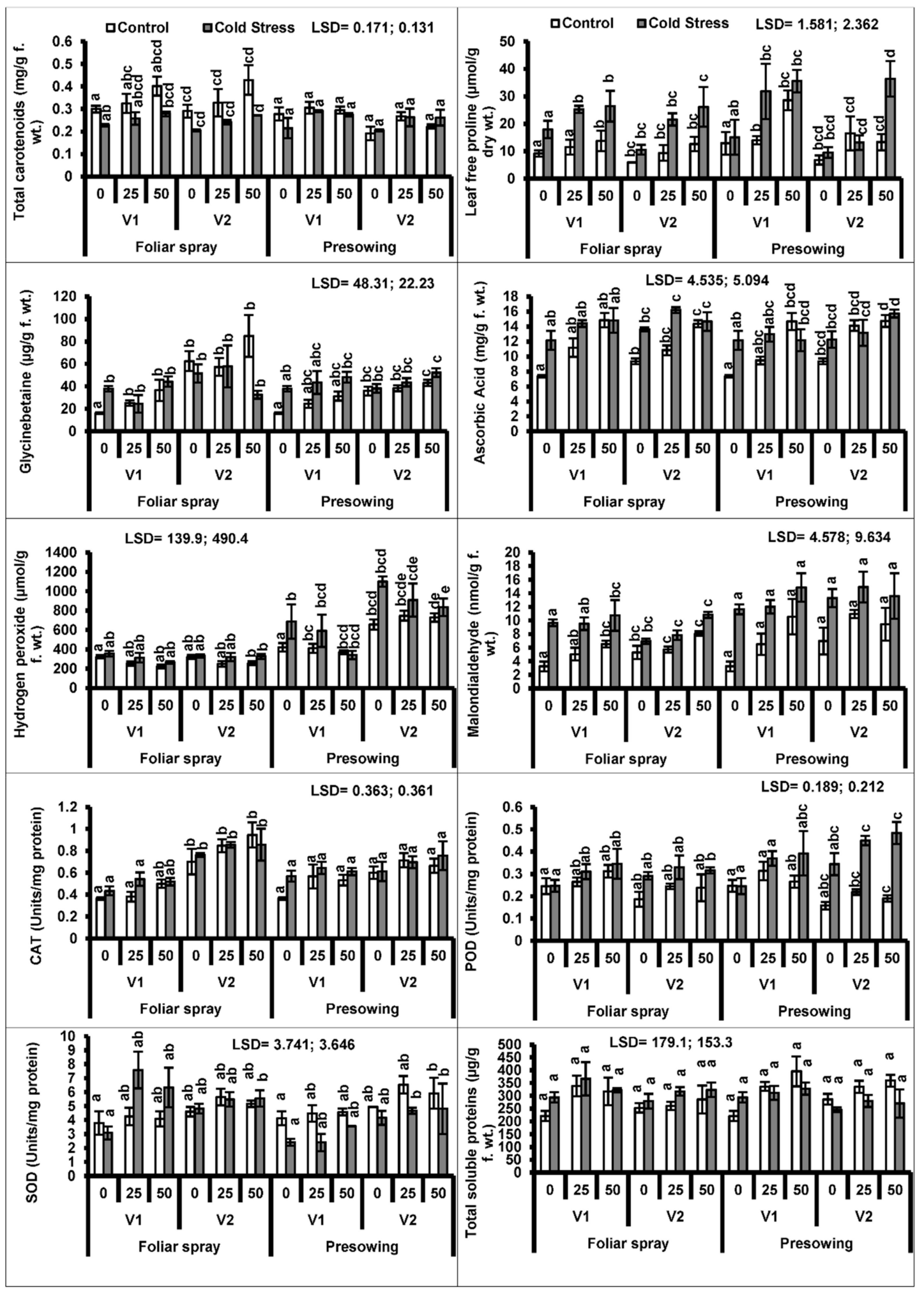

Figure 2. Total carotenoids, proline, glycine betaine, ascorbic acid, hydrogen peroxide, malondialdehyde contents, activities of enzymatic antioxidants (catalase (CAT), peroxidase (POD) and SOD) and total soluble proteins of quinoa plants subjected to chilling stress (mean \pm S.E.); Different letters (a-e) show significant differences among treatments. f. wt; fresh weight. 

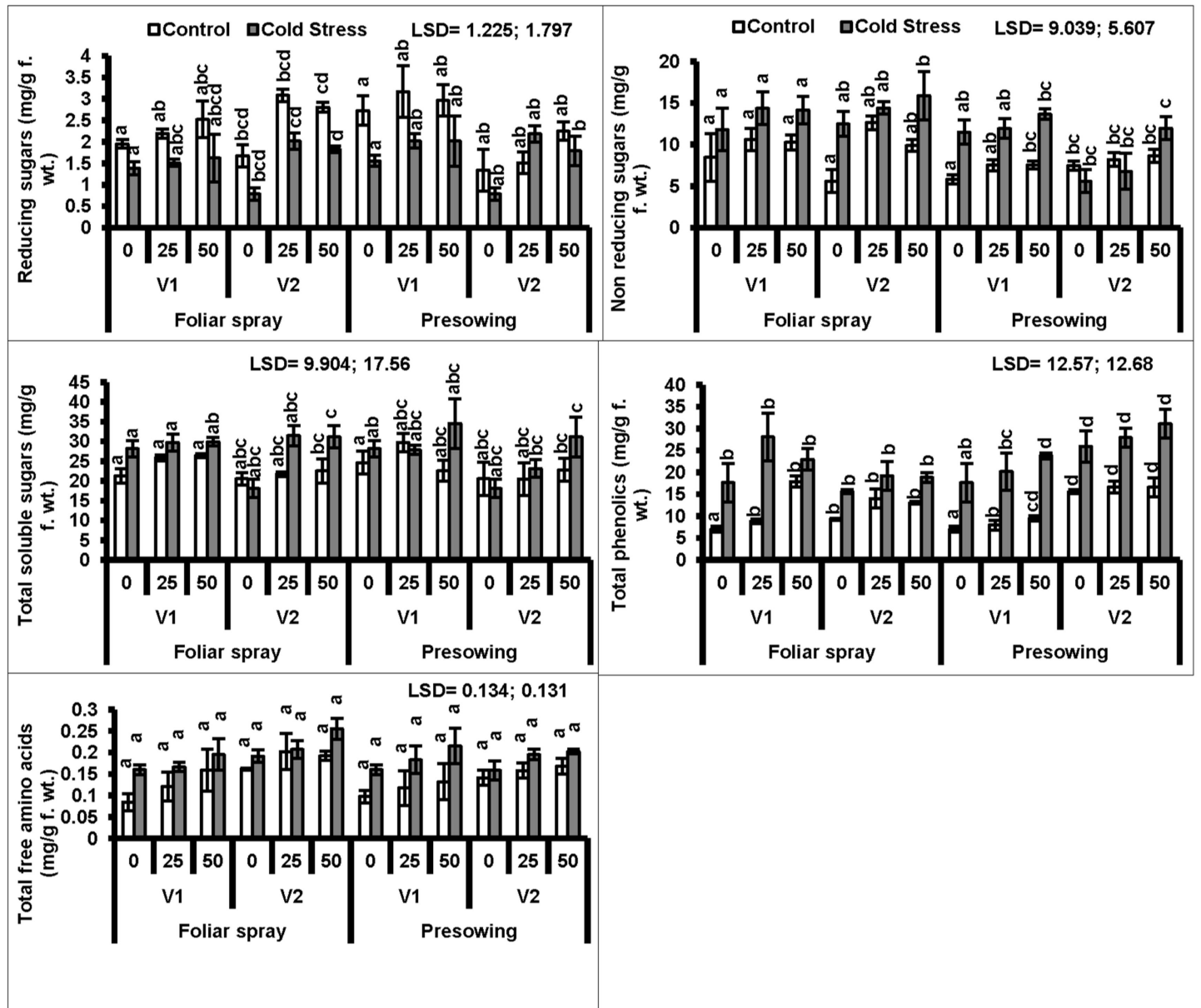

Figure 3. Reducing sugars, non-reducing sugars, total soluble sugars, total phenolics and total free amino acids of quinoa plants subjected to chilling stress (mean \pm S.E.); Different letters (a-d) show significant differences among treatments. $\mathrm{f}$. wt; fresh weight.

A high accumulation of total soluble sugars was observed in the plants of both quinoa cultivars raised from cold-stressed seeds. Cultivar $\mathrm{V}_{1}$ showed a uniform response in sugar accumulation to both foliar and pre-sowing proline treatments, whereas foliar treatment gave more significant results in cv. $\mathrm{V}_{2}$ as compared to the other cultivar (Figure 3).

A marked increase in total free amino acids in both quinoa cultivars was observed due to low-temperature stress applied as seed treatment. However, foliar and pre-sowing proline applications significantly $(P \leq 0.01)$ increased the total free amino acids in both quinoa cultivars. Cultivar $\mathrm{V}_{2}$ was better at the rate of $50 \mathrm{mM}$ proline applied as a foliage spray, whereas cultivar $\mathrm{V}_{1}$ accumulated higher amounts of amino acids at $50 \mathrm{mM}$ proline applied as a pre-sowing treatment (Figure 3).

\section{Discussion}

Biochemical analyses of plants under a variety of abiotic stresses reflect the fact that plants accumulate compatible metabolites significantly, which effectively protects the plants under stress $[47,48]$. As compared to the other osmoprotectants, proline accumulates in sufficient amounts and interferes with the plant defensive metabolic system by scavenging reactive oxygen species so as to improve plant growth and metabolism [16]. The stress-sensitive plants incapable of producing this amino acid remain usually intolerant to ecological stresses [16]. Similarly, it is evident that the exogenous use of proline provides relief against stress injuries [36]. It has been observed that treatment of this amino acid not only enhanced adaptive plant metabolism but also accelerated nitrogen absorption and its assimilation [49]. In the present study, the shoot fresh and dry weights of quinoa 
plants decreased significantly, but no significant change was observed on the root fresh and dry weights of plants raised from cold-stressed seeds. Both modes (foliar and pre-sowing) of proline application significantly improved the shoot and root fresh and dry weights of both quinoa plants. Similarly, in the case of plant height, low-temperature stress applied as a seed treatment significantly reduced shoot length, but it did not affect the root length. Both modes of proline application significantly enhanced the shoot and root lengths of quinoa plants raised from low-temperature treated seeds. Similar effects on growth attributes have already been reported in maize [25], quinoa [50] and faba bean [51] under drought stress, rice [52] and fenugreek plant [53] under salinity stress. These findings show that the application of proline ameliorates the adverse effects of abiotic stresses, perhaps by modulating cell division and cell elongation so as to produce a high plant biomass and vigor. Exogenously applied proline acts as an important nutrient, which could be beneficial for stress tolerance as well as enhancing the activities/levels of enzymatic and non-enzymatic antioxidants [54,55].

Prolonged stress conditions are believed to cause an impairment in the accumulation of photosynthetic pigments such as chlorophyll in plants [56], as a result of which photosynthetic events such as the electron transport chain and conversion of light energy into metabolic energy undergo alteration $[57,58]$. In the current study, plants raised from cold-treated seeds showed reduced chlorophyll $a$ and $b$, total chlorophyll, and carotenoids in both quinoa cultivars, whereas the application of proline significantly improved all these pigments. These results are in line with those of Elewa, et al. [50], in which an increase in chlorophyll pigments was observed due to proline spray at the rate of 12.5 and $25 \mathrm{mM}$ to quinoa plants exposed to drought stress. A reduction in photosynthetic pigments could have been due to stress-induced impairment in pigment synthesis [59]. In sunflower plants, Sadak and Mostafa [60] found a significant enhancement in chlorophyll $a$ and $b$ and carotenoids due to proline (2.5. 5.0 and $7.5 \mathrm{mM}$ ) application as a pre-sowing treatment under drought stress. Similar findings were reported by Ali, et al. [51] in faba bean plants and Hasanuzzaman, et al. [61] in rice plants under salt stress. The degradation of chloroplast and photosynthetic pigments due to stress conditions has a severe impact on the accumulation of carbon assimilates [51].

Under stress conditions, plants counteract the stress-induced adversaries by accumulating compatible osmoprotectants such as glycine betaine and proline etc., which play a key role in regulating osmotica accumulation in plants [19]. The external use of such potential protectants ameliorates the adverse effects of oxidative stress by activating the antioxidant defense system and the maintenance of osmotic homeostasis, turgor potential, and water relations [62]. Likewise, in the present study, chilling stress applied as a seed treatment increased endogenous concentrations of proline and glycine betaine in both quinoa cultivars. The exogenous application of proline also posed a positive effect in enhancing the accumulation of both these osmoprotectants in plants raised from cold-treated seeds. These findings are consistent with those of Abd Elhamid, et al. [53], in which the endogenous level of proline was found to be improved upon proline external application in saline-stressed Phaseolus vulgaris. In barley seedlings, exogenous application of proline improved in vivo levels of proline [63], whereas in rice plants, intracellular proline levels were increased upon a topical spray of proline $(5 \mathrm{mM})$ under saline stress [61]. In view of the above results, it can be suggested that proline is an effective protectant to make plants robust against abiotic stress adversity.

Ascorbic acid acts as an antioxidant, which can preserve plant photosynthesis and aerobic metabolic activities, which are otherwise deteriorated during oxidative stress [64]. Chilling stress applied as a pre-sowing seed treatment caused a significant increase in ascorbic acid (AsA) concentration in both quinoa cultivars. However, foliar-applied proline proved to be more efficient in promoting ascorbic acid levels than the seed priming treatment in quinoa plants. Earlier, Sakr, et al. [65] reported that a foliar spray of proline at $200 \mathrm{mg} \mathrm{L}^{-1}$ enhanced the levels of ascorbic acid under salinity stress in canola plants. Similarly, Abdelhamid, et al. [66] reported that the treatment of $5.0 \mathrm{mM}$ proline elevated AsA levels in Phaseolus vulgaris under saline stress

Plants exposed to stressful environments experience enhanced lipid peroxidation and malondialdehyde contents. High MDA contents are generally considered to be a potential indicator of 
oxidative stress in plants $[67,68]$. High production of $\mathrm{H}_{2} \mathrm{O}_{2}$ as well as MDA is also one of the toxic effects of oxidative stress [69]. In the present study, chilling stress applied as a pre-sowing treatment had a significant effect on $\mathrm{H}_{2} \mathrm{O}_{2}$ concentration and levels of MDA in both quinoa cultivars. Both foliar and pre-sowing treatments of proline did not play a significant role in lowering $\mathrm{H}_{2} \mathrm{O}_{2}$ levels, but they reduced the MDA contents in both quinoa cultivars. Addressing this concern, a previous study by Hasanuzzaman, et al. [61] showed that an external application of proline reduced $\mathrm{H}_{2} \mathrm{O}_{2}$ and MDA levels in rice plants under salinity stress by lowering the oxidative defense system. These results are in agreement with those of Ali, et al. [51], in which it was reported that drought stress enhanced the malondialdehyde in faba bean plants. Generally, it is believed that proline treatment lowers the production of free radicals and subsequently retards lipid peroxidation [36,70].

Plants upon exposure to stress conditions usually experience adaptive physiological changes. Under adverse stress conditions, the accumulation of antioxidants is an important phenomenon. High activities of antioxidant enzymes such as catalase, superoxide dismutase and peroxidase play an important role in ameliorating stress injuries caused by reactive oxygen species [71]. The present study showed that cold-stress conditions applied as a pre-sowing seed treatment significantly increased the activities of peroxidase enzyme but remained non-significant for catalase and superoxide dismutase activities. The treatments of proline as seed soaking and as a foliar spray improved the activities of CAT, POD and SOD in plants of both quinoa cultivars raised from low-temperature treated seeds, which indicated that proline may be involved in the oxidative defense system. These findings are in line with some previous reports conducted under different abiotic stresses; e.g., Osman [71] reported that the application of proline to pea plants under drought stress showed improved activities of catalase and ascorbate peroxidase. Similar results were reported by Elewa, et al. [50] in quinoa, faba bean [51], and barley [63]. The enhancement of antioxidant enzymes could be effective in improving the growth and development of most plants under stress conditions [72].

The intrinsic total soluble phenolics increased in quinoa plants in response to cold stress applied as a seed treatment. The external application of proline was observed to be effective in further improving the phenolic levels. These results are in agreement with those of Elewa, et al. [50] in quinoa and Abd Elhamid, et al. [53] in tomato. It could be possible that phenolics function in conjunction with other potential antioxidants to counteract ROS [73].

To survive under stress, the accumulation of osmoregulators as well as oxidative scavengers are important defensive features of plants. In this study, cold stress applied as a pre-sowing treatment caused a marked accumulation of total soluble sugars in plants of both quinoa cultivars. Overall, both modes of proline application significantly improved total soluble sugars in plants raised from seeds treated with cold. In contrast, Ben Ahmed, et al. [74] reported a decrease in sugar levels in proline-treated olive plants under salinity stress. Recently, Zali and Ehsanzadeh [75] have reported an enhanced accumulation of sugars in fennel plants exposed to drought stress. It is believed that sugar levels during stress may neutralize the reactive oxygen species and help maintain the turgidity of cells and the integrity of cellular membranes [76].

\section{Conclusions}

In conclusion, chilling stress adversely affected the plant growth and metabolism of quinoa plants. Furthermore, the exogenous application of proline was found to be beneficial for improving growth, chlorophyll $a$, carotenoids, glycine betaine, ascorbic acid, the activities of antioxidant enzymes, and in lowering the hydrogen peroxide and malondialdehyde contents in both quinoa cultivars. As a whole, the exogenous application of proline as a foliar spray or seed treatment were more beneficial than the other treatments used.

Supplementary Materials: The following are available online at http://www.mdpi.com/2223-7747/8/12/588/s1, Figure S1: Overview of both quinoa cultivars V1 (A) and V2 (B) subjected to exogenously applied proline and cold stress conditions. 
Author Contributions: Conceptualization, N.A.A., S.I., M.A. and P.A.; methodology, H.Y.; software, M.N.A. and L.W.; validation, N.A.A., M.N.A. and P.A.; formal analysis, N.K., L.W. and M.S.; investigation, H.Y.; resources, N.A.A.; data curation, N.A.A. and M.A.; writing-original draft preparation, H.Y., S.I., N.K. and M.S.; writing-review and editing, N.A.A., M.A. and P.A.; visualization, N.A.A.; supervision, N.A.A.; project administration, N.A.A.; funding acquisition, M.N.A., L.W. and P.A.

Funding: RSP-2019/116.

Acknowledgments: The authors would like to extend their sincere appreciation to the Researchers Supporting Project Number (RSP-2019/116), King Saud University, Riyadh, Saudi Arabia.

Conflicts of Interest: The authors declare no conflict of interest.

\section{References}

1. Shahbaz, M.; Ashraf, M.; Al-Qurainy, F.; Harris, P.J.C. Salt tolerance in selected vegetable crops. Crit. Rev. Plant Sci. 2012, 31, 303-320. [CrossRef]

2. Roychoudhury, A.; Basu, S.; Sengupta, D.N. Antioxidants and stress-related metabolites in the seedlings of two indica rice varieties exposed to cadmium chloride toxicity. Acta Physiol. Plant. 2011, 34, 835-847. [CrossRef]

3. Shahbaz, M.; Ashraf, M. Improving salinity tolerance in cereals. Crit. Rev. Plant Sci. 2013, 32, $237-249$. [CrossRef]

4. Blokhina, O. Antioxidants, oxidative damage and oxygen deprivation stress: A review. Ann. Bot. 2003, 91, 179-194. [CrossRef] [PubMed]

5. Hossain, M.A.; Bhattacharjee, S.; Armin, S.-M.; Qian, P.; Xin, W.; Li, H.-Y.; Burritt, D.J.; Fujita, M.; Tran, L.-S.P. Hydrogen peroxide priming modulates abiotic oxidative stress tolerance: insights from ROS detoxification and scavenging. Front. Plant Sci. 2015, 6. [CrossRef]

6. Schwartz, M.D.; Ahas, R.; Aasa, A. Onset of spring starting earlier across the Northern Hemisphere. Global Change Biol. 2006, 12, 343-351. [CrossRef]

7. Ahmad, T.; Shahzad, J.e.S. Low temperature stress effect on wheat cultivars germination. Afr. J. Microbiol. Res. 2012, 6, 1265-1269. [CrossRef]

8. Ghadirnezhad, R.; Fallah, A. Temperature effect on yield and yield components of different rice cultivars in flowering stage. Int. J. Agron. 2014, 2014, 1-4. [CrossRef]

9. Clayton, S.; Neves, P.C. Country snapshot: Brazil. Rice Today 2011, 10, 16-17.

10. De Lima, J.C.; Loss-Morais, G.; Margis, R. MicroRNAs play critical roles during plant development and in response to abiotic stresses. Genet. Mol. Biol. 2012, 35, 1069-1077. [CrossRef]

11. Shinozaki, K.; Yamaguchi-Shinozaki, K.; Seki, M. Regulatory network of gene expression in the drought and cold stress responses. Curr. Opin. Plant Biol. 2003, 6, 410-417. [CrossRef]

12. Sanghera, G.; Wani, S.; Hussain, W.; Singh, N. Engineering cold stress tolerance in crop plants. Curr. Genom. 2011, 12, 30-43. [CrossRef] [PubMed]

13. Chen, Z.; Sun, L.; Liu, P.; Liu, G.; Tian, J.; Liao, H. Malate synthesis and secretion mediated by a manganese-enhanced malate dehydrogenase confers superior manganese tolerance in Stylosanthes guianensis. Plant Physiol. 2015, 167, 176-188. [CrossRef] [PubMed]

14. Ashraf, M.; Foolad, M.R. Crop breeding for salt tolerance in the era of molecular markers and marker-assisted selection. Plant Breed. 2013, 132, 10-20. [CrossRef]

15. Wu, D.; Cai, S.; Chen, M.; Ye, L.; Chen, Z.; Zhang, H.; Dai, F.; Wu, F.; Zhang, G. Tissue metabolic responses to salt stress in wild and cultivated barley. PLoS ONE 2013, 8, e55431. [CrossRef]

16. Ashraf, M.; Foolad, M.R. Roles of glycine betaine and proline in improving plant abiotic stress resistance. Environ. Exp. Bot. 2007, 59, 206-216. [CrossRef]

17. Soshinkova, T.N.; Radyukina, N.L.; Korolkova, D.V.; Nosov, A.V. Proline and functioning of the antioxidant system in Thellungiella salsuginea plants and cultured cells subjected to oxidative stress. Russ. J. Plant Physiol. 2013, 60, 41-54. [CrossRef]

18. Molla, M.R.; Ali, M.R.; Hasanuzzaman, M.; Al-Mamun, M.H.; Ahmed, A.; Nazim-Ud-Dowla, M.A.N.; Rohman, M.M. Exogenous proline and betaine-induced upregulation of glutathione transferase and glyoxalase I in lentil (Lens culinaris) under drought stress. Not. Bot. Hort. Agrobot. Cluj-Napoca 2014, 42. [CrossRef] 
19. Szabados, L.; Savouré, A. Proline: A multifunctional amino acid. Trends Plant Sci. 2010, 15, 89-97. [CrossRef]

20. Giri, J. Glycinebetaine and abiotic stress tolerance in plants. Plant Signal. Behav. 2011, 6, 1746-1751. [CrossRef]

21. Kumar, S.; Nayyar, H.; Bhanwara, R.; Upadhyaya, H. Chilling stress effects on reproductive biology of chickpea. J. SAT Agric. Res. 2010, 8, 1-14.

22. Mattioli, R.; Marchese, D.; D'Angeli, S.; Altamura, M.M.; Costantino, P.; Trovato, M. Modulation of intracellular proline levels affects flowering time and inflorescence architecture in Arabidopsis. Plant Mol. Biol. 2008, 66, 277-288. [CrossRef] [PubMed]

23. Funck, D.; Winter, G.; Baumgarten, L.; Forlani, G. Requirement of proline synthesis during Arabidopsis reproductive development. BMC Plant Biol. 2012, 12, 191. [CrossRef] [PubMed]

24. Lehmann, S.; Funck, D.; Szabados, L.; Rentsch, D. Proline metabolism and transport in plant development. Amino Acids 2010, 39, 949-962. [CrossRef] [PubMed]

25. Ali, Q.; Ashraf, M.; Athar, H.U.R. Exogenously applied proline at different growth stages enhances growth of two maize cultivars grown under water deficit conditions. Pak. J. Bot. 2007, 39, 1133-1144.

26. Okuma, E.; Murakami, Y.; Shimoishi, Y.; Tada, M.; Murata, Y. Effects of exogenous application of proline and betaine on the growth of tobacco cultured cells under saline conditions. Soil Sci. Plant Nutr. 2004, 50, 1301-1305. [CrossRef]

27. Talat, A.; Nawaz, K.; Hussian, K.; Bhatti, K.H.; Siddiqi, E.H.; Khalid, A.; Anwer, S.; Sharif, M.U. Foliar application of proline for salt tolerance of two wheat (Triticum aestivum L.) cultivars. World Appl. Sci. J. 2013, 22, 547-554.

28. Ben Ahmed, C.; Magdich, S.; Ben Rouina, B.; Sensoy, S.; Boukhris, M.; Ben Abdullah, F. Exogenous proline effects on water relations and ions contents in leaves and roots of young olive. Amino Acids 2010, 40, 565-573. [CrossRef]

29. Vega-Gálvez, A.; Miranda, M.; Vergara, J.; Uribe, E.; Puente, L.; Martínez, E.A. Nutrition facts and functional potential of quinoa (Chenopodium quinoa willd.), an ancient Andean grain: a review. J. Sci. Food Agric. 2010, 90, 2541-2547. [CrossRef]

30. Stikic, R.; Glamoclija, D.; Demin, M.; Vucelic-Radovic, B.; Jovanovic, Z.; Milojkovic-Opsenica, D.; Jacobsen, S.-E.; Milovanovic, M. Agronomical and nutritional evaluation of quinoa seeds (Chenopodium quinoa Willd.) as an ingredient in bread formulations. J. Cereal Sci. 2012, 55, 132-138. [CrossRef]

31. Jacobsen, S.E. The worldwide potential for quinoa (Chenopodium quinoa Willd.). Food Rev. Int. 2003, 19, 167-177. [CrossRef]

32. Jacobsen, S.E. The situation for quinoa and its production in Southern Bolivia: From economic success to environmental disaster. J. Agron. Crop. Sci. 2011, 197, 390-399. [CrossRef]

33. Ruiz, K.B.; Biondi, S.; Martínez, E.A.; Orsini, F.; Antognoni, F.; Jacobsen, S.E. Quinoa-A model crop for understanding salt-tolerance mechanisms in halophytes. Plant Biosys. 2015, 150, 357-371. [CrossRef]

34. Repo-Carrasco, R.; Espinoza, C.; Jacobsen, S.E. Nutritional value and use of the Andean crops quinoa (Chenopodium quinoa) and Kañiwa (Chenopodium pallidicaule). Food Rev. Int. 2003, 19, 179-189. [CrossRef]

35. Llorente, J. Quinoa. Available online: http://dsalud.com/index.php.

36. Kamran, M.; Shahbaz, M.; Ashraf, M.; Akram, N.A. Alleviation of drought-induced adverse effects in spring wheat (Triticum aestivum L.) using proline as a pre-sowing seed treatment. Pak. J. Bot. 2009, 41, 621-632.

37. Arnon, D.I. Copper enzymes in isolated chloroplasts, polyphenoxidase in Beta vulgaris. Plant Physiol. 1949, 24, 1-15. [CrossRef] [PubMed]

38. Velikova, V.; Yordanov, I.; Edreva, A. Oxidative stress and some antioxidant systems in acid rain-treated bean plants. Plant Sci. 2000, 151, 59-66. [CrossRef]

39. Heath, R.L.; Packer, L. Photoperoxidation in isolated chloroplasts. Arch. Biochem. Biophys. 1968, 125, 189-198. [CrossRef]

40. Bates, L.S.; Waldren, R.P.; Teare, I.D. Rapid determination of free proline for water-stress studies. Plant Soil 1973, 39, 205-207. [CrossRef]

41. Mukherjee, S.P.; Choudhuri, M.A. Implications of water stress-induced changes in the levels of endogenous ascorbic acid and hydrogen peroxide in Vigna seedlings. Physiol. Plant. 1983, 58, 166-170. [CrossRef]

42. Chance, B.; Maehly, A.C. Assay of catalases and peroxidases. Meth. Enzymol. 1955, 2, 764-775. [CrossRef]

43. Giannopolitis, C.N.; Ries, S.K. Superoxide dismutases. Plant Physiol. 1977, 59, 309-314. [CrossRef] [PubMed]

44. Yemm, E.W.; Willis, A.J. The estimation of carbohydrates in plant extracts by anthrone. Biochem. J. 1954, 57, 508-514. [CrossRef] [PubMed] 
45. Julkunen-Tiitto, R. Phenolic constituents in the leaves of northern willows: Methods for the analysis of certain phenolics. J. Agric. Food Chem. 1985, 33, 213-217. [CrossRef]

46. Hamilton, P.B.; Van Slyke, D.D.; Lemish, S. The gasometric determination of free amino acids in blood filtrates by the ninhydrin-carbon dioxide method. J. Biol. Chem. 1943, 150, 231-250.

47. Kahlaoui, B.; Hachicha, M.; Misle, E.; Fidalgo, F.; Teixeira, J. Physiological and biochemical responses to the exogenous application of proline of tomato plants irrigated with saline water. J. Saudi Soc. Agric. Sci. 2018, 17, 17-23. [CrossRef]

48. Akram, N.A.; Shafiq, F.; Ashraf, M. Ascorbic acid-a potential oxidant scavenger and its role in plant development and abiotic stress tolerance. Front. Plant Sci. 2017, 8. [CrossRef]

49. Cerdán, M.; Sánchez-Sánchez, A.; Jordá, J.D.; Juárez, M.; Sánchez-Andreu, J. Effect of commercial amino acids on iron nutrition of tomato plants grown under lime-induced iron deficiency. J. Plant Nutr. Soil Sci. 2013, 176, 859-866. [CrossRef]

50. Elewa, T.A.; Sadak, M.S.; Saad, A.M. Proline treatment improves physiological responses in quinoa plants under drought stress. Biosci. Res. 2017, 14, 21-33.

51. Ali, H.; Siddiqui, M.; Al-Whaibi, M.; Basalah, M.; Sakran, A.; El-Zaidy, M. Effect of proline and abscisic acid on the growth and physiological performance of faba bean under water stress. Pak. J. Bot. 2013, 45, 933-940.

52. Deivanai, S.; Xavier, R.; Vinod, V.; Timalata, K.; Lim, O. Role of exogenous proline in ameliorating salt stress at early stage in two rice cultivars. J. Stress Physiol. Biochem. 2011, 7.

53. Abd Elhamid, E.M.; Sadak, M.S.; Tawfik, M. Physiological response of Fenugreek plant to the application of proline under different water regimes. Res. J. Pharmaceut. Biol. Chem. Sci. 2016, 7, 580-594.

54. Yan, Z.; Guo, S.; Shu, S.; Sun, J.; Tezuka, T. Effects of proline on photosynthesis, root reactive oxygen species (ROS) metabolism in two melon cultivars (Cucumis melo L.) under $\mathrm{NaCl}$ stress. Afr. J. Biotechnol. 2011, 10, 18381-18390. [CrossRef]

55. Çelik, Ö.; Ayan, A.; Atak, Ç. Enzymatic and non-enzymatic comparison of two different industrial tomato (Solanum lycopersicum) varieties against drought stress. Bot. Stud. 2017, 58. [CrossRef] [PubMed]

56. Ashraf, M.; Harris, P.J.C. Photosynthesis under stressful environments: An overview. Photosynthetica 2013, 51, 163-190. [CrossRef]

57. Massacci, A.; Nabiev, S.M.; Pietrosanti, L.; Nematov, S.K.; Chernikova, T.N.; Thor, K.; Leipner, J. Response of the photosynthetic apparatus of cotton (Gossypium hirsutum) to the onset of drought stress under field conditions studied by gas-exchange analysis and chlorophyll fluorescence imaging. Plant Physiol. Biochem. 2008, 46, 189-195. [CrossRef]

58. Smolikova, G.N.; Laman, N.A.; Boriskevich, O.V. Role of chlorophylls and carotenoids in seed tolerance to abiotic stressors. Russ. J. Plant Physiol. 2011, 58, 965-973. [CrossRef]

59. Pandey, H.; Baig, M.; Bhatt, R. Effect of moisture stress on chlorophyll accumulation and nitrate reductase activity at vegetative and flowering stage in Avena species. Agric. Sci. Res. J. 2012, 2, 111-118.

60. Sadak, M.S.; Mostafa, H.A. Physiological role of pre-sowing seed with proline on some growth, biochemical aspects, yield quantity and quality of two sunflower cultivars grown under seawater salinity stress. Sci. Agric. 2015, 9, 60-69.

61. Hasanuzzaman, M.; Alam, M.M.; Rahman, A.; Hasanuzzaman, M.; Nahar, K.; Fujita, M. Exogenous proline and glycine betaine mediated upregulation of antioxidant defense and glyoxalase systems provides better protection against salt-induced oxidative stress in two rice (Oryza sativa L.) varieties. BioMed Res. Int. 2014, 2014, 1-17. [CrossRef]

62. Nawaz, K.; Ashraf, M. Exogenous application of glycinebetaine modulates activities of antioxidants in maize plants subjected to salt stress. J. Agron. Crop. Sci. 2010, 196, 28-37. [CrossRef]

63. Agami, R.A. Applications of ascorbic acid or proline increase resistance to salt stress in barley seedlings. Biol. Plant. 2014, 58, 341-347. [CrossRef]

64. Mazid, M.; Khan, T.A.; Khan, Z.H.; Quddusi, S.; Mohammad, F. Occurrence, biosynthesis and potentialities of ascorbic acid in plants. Int. J. Plant Anim. Environ. Sci. 2011, 1, 167-184.

65. Sakr, M.T.; El-Sarkassy, N.M.; Fuller, M.P. Osmoregulators proline and glycine betaine counteract salinity stress in canola. Agron. Sustain. Dev. 2012, 32, 747-754. [CrossRef]

66. Abdelhamid, M.T.; Rady, M.M.; Osman, A.S.; Abdalla, M.A. Exogenous application of proline alleviates salt-induced oxidative stress in Phaseolus vulgaris L. plants. J. Hort. Sci. Biotechnol. 2013, 88, 439-446. [CrossRef] 
67. Ashraf, M. Biotechnological approach of improving plant salt tolerance using antioxidants as markers. Biotechnol. Adv. 2009, 27, 84-93. [CrossRef]

68. Garg, N.; Manchanda, G. ROS generation in plants: Boon or bane? Plant Biosyst. 2009, 143, 81-96. [CrossRef]

69. Hasanuzzaman, M.; Hossain, M.A.; Fujita, M. Nitric oxide modulates antioxidant defense and the methylglyoxal detoxification system and reduces salinity-induced damage of wheat seedlings. Plant Biotechnol. Rep. 2011, 5, 353-365. [CrossRef]

70. Siddiqui, M.H.; Al-Whaibi, M.H.; Sakran, A.M.; Ali, H.M.; Basalah, M.O.; Faisal, M.; Alatar, A.; Al-Amri, A.A. Calcium-induced amelioration of boron toxicity in radish. J. Plant Growth Regul. 2012, 32, 61-71. [CrossRef]

71. Osman, H.S. Enhancing antioxidant-yield relationship of pea plant under drought at different growth stages by exogenously applied glycine betaine and proline. Ann. Agric. Sci. 2015, 60, 389-402. [CrossRef]

72. Azevedo, R.A.; Gratão, P.L.; Monteiro, C.C.; Carvalho, R.F. What is new in the research on cadmium-induced stress in plants? Food Energy Secur. 2012, 1, 133-140. [CrossRef]

73. Sgherri, C.; Cosi, E.; Navari-Izzo, F. Phenols and antioxidative status of Raphanus sativus grown in copper excess. Physiol. Plant. 2003, 118, 21-28. [CrossRef] [PubMed]

74. Ben Ahmed, C.; Ben Rouina, B.; Sensoy, S.; Boukhriss, M.; Ben Abdullah, F. Exogenous proline effects on photosynthetic performance and antioxidant defense system of young olive tree. J. Agric. Food Chem. 2010, 58, 4216-4222. [CrossRef] [PubMed]

75. Zali, G.A.; Ehsanzadeh, P. Exogenous proline improves osmoregulation, physiological functions, essential oil, and seed yield of fennel. Ind. Crops Prod. 2018, 111, 133-140. [CrossRef]

76. Hosseini, S.M.; Hasanloo, T.; Mohammadi, S. Physiological characteristics, antioxidant enzyme activities, and gene expression in 2 spring canola (Brassica napus L.) cultivars under drought stress conditions. Turk. J. Agric. Forest. 2014, 39, 413-420. [CrossRef]

(C) 2019 by the authors. Licensee MDPI, Basel, Switzerland. This article is an open access article distributed under the terms and conditions of the Creative Commons Attribution (CC BY) license (http://creativecommons.org/licenses/by/4.0/). 